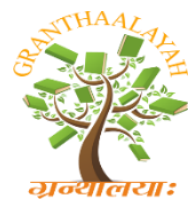

\author{
INTERNATIONAL JOURNAL OF RESEARCH - \\ GRANTHAALAYAH \\ A knowledge Repository
}

Science

\title{
SOME INCLUSION PROPERTIES FOR CERTAIN K-UNIFORMLY SUBCLASSES OF ANALYTIC FUNCTIONS ASSOCIATED WITH WRIGHT FUNCTION
}

\author{
E. E. Ali *1 \\ ${ }^{* 1}$ Department of Mathematics and Computer Science, Faculty of Science, Port Said University, \\ Port Said 42521, Egypt
}

\begin{abstract}
A new operator $\mathbf{W}_{\lambda, \mu}^{\alpha} f(z)=z+\sum_{n \geq 2} \frac{4^{n-1}(\alpha)_{(n-1)} \Gamma(\lambda(n-1)+\mu)}{\Gamma(\mu)} a_{n} z^{n}$ is introduced for functions of the form $f(z)=z+\sum_{n=2}^{\infty} a_{n} z^{n}$ which are analytic in the open unit disk $\mathbf{U}=\{z \in \mathrm{C}:|z|<1\}$. We introduce several inclusion properties of the new $k$-uniformly classes $U S^{*}(\alpha ; k ; \gamma), U C(\alpha ; k ; \gamma)$, $U K(\alpha ; k ; \gamma, \beta)$ and $U K^{*}(\alpha ; k ; \gamma, \beta)$ of analytic functions defined by using the Wright function with the operator $\mathbf{W}_{\lambda, \mu}^{\alpha}$ and the main object of this paper is to investigate various inclusion relationships for these classes. In addition, we proved that a special property is preserved by some integral operators.
\end{abstract}

Keywords: Analytic Functions; K-Uniformly Starlike Functions; K-Uniformly Convex Functions; K-Uniformly Close-To-Convex Functions; K-Uniformly Quasi-Convex Functions; Hadamard Product; Subordination.

2000 Mathematics Subject Classification: 30C45; 30D30; 33D20.

Cite This Article: E. E. Ali. (2019). "SOME INCLUSION PROPERTIES FOR CERTAIN KUNIFORMLY SUBCLASSES OF ANALYTIC FUNCTIONS ASSOCIATED WITH WRIGHT FUNCTION." International Journal of Research - Granthaalayah, 7(9), 218-229. https://doi.org/10.29121/granthaalayah.v7.i9.2019.604.

\section{Introduction}

Let $\mathrm{A}$ denote the class of functions of the form

$$
f(z)=z+\sum_{n=2}^{\infty} a_{n} z^{n}
$$

Which are analytic in the open unit disk $\mathbf{U}=\{z \in \mathrm{C}:|z|<1\}$. If $\boldsymbol{I}$ and $\boldsymbol{g}$ are analytic in $\mathbf{U}$, we say that $f$ is subordinate to $g$, written $f \prec g$ or $f(z) \prec g(z)$, if there exists a Schwarz 
function $\omega$, analytic in $\mathbf{U}$ with $\omega(0)=0$ and $|\omega(z)|<1 \quad(z \in \mathbf{U})$, such that $f(z)=g(\omega(z))$ $(z \in \mathbf{U})$. In particular, if the function $g$ is univalent in $\mathbf{U}$, the above subordination is equivalent to $f(0)=g(0)$ and $f(\mathbf{U}) \subset g(\mathbf{U}) \quad$ (see [9] and [10]).

For functions $f(z) \in \mathbf{A}$, given by (1.1), and $g(z) \in \mathbf{A}$ defined by

$$
g(z)=z+\sum_{k=2}^{\infty} b_{k} z^{k}
$$

then the Hadamard product (or convolution) of $f(z)$ and $g(z)$ is given by

$$
(f * g)(z)=z+\sum_{k=2}^{\infty} a_{k} b_{k} z^{k}=(g * f)(z) \quad(z \in U)
$$

For $0 \leq \gamma, \beta<1$, we denote by $S^{*}(\gamma), C(\gamma), K(\gamma, \beta)$ and $K^{*}(\gamma, \beta)$ the subclasses of $\mathbf{A}$ consisting of all analytic functions which are, respectively, starlike of order $\gamma$, convex of order $\gamma$, close-to-convex of order $\gamma$, and type $\beta$ and quasi-convex of order $\gamma$, and type $\beta$ in $\mathbf{U}$.

Now, we introduce the subclasses $U S^{*}(k ; \gamma), U C(k ; \gamma), U K(k ; \gamma, \beta)$ and $U K^{*}(k ; \gamma, \beta)$ of the class $\mathbf{A}$ for $0 \leq \gamma, \beta<1$, and $k \geq 0$, which are defined by

$$
\begin{gathered}
U S^{*}(k ; \gamma)=\left\{f \in \mathbf{A}: \mathfrak{R}\left(\frac{z f^{\prime}(z)}{f(z)}-\gamma\right)>k \mid \frac{z f^{\prime}(z)}{f(z)}-1\right\}, \\
U C(k ; \gamma)=\left\{f \in \mathbf{A}: \mathfrak{R}\left(1+\frac{z f^{\prime \prime}(z)}{f^{\prime}(z)}-\gamma\right)>k\left|\frac{z f^{\prime \prime}(z)}{f^{\prime}(z)}\right|+\gamma\right\}, \\
U K(k ; \gamma, \beta)=\left\{f \in \mathbf{A}: \exists g \in U S^{*}(k ; \beta) \text { s.t. } \mathfrak{R}\left(\frac{z f^{\prime}(z)}{g(z)}-\gamma\right)>k\left|\frac{z f^{\prime}(z)}{g(z)}-1\right|\right\}, \\
U K^{*}(k ; \gamma, \beta)=\left\{f \in \mathbf{A}: \exists g \in U C(k ; \gamma) \text { s.t.R }\left(\frac{\left(z f^{\prime}(z)\right)^{\prime}}{g{ }^{\prime}(z)}-\gamma\right)>k\left|\frac{\left(z f^{\prime}(z)\right)^{\prime}}{g{ }^{\prime}(z)}-1\right|\right\} .
\end{gathered}
$$

We note that

$$
\begin{gathered}
U S^{*}(0 ; \gamma)=S^{*}(k ; \gamma), U C(0 ; \gamma)=C(\gamma), \\
U K(0 ; \gamma, \beta)=K(\gamma, \beta), U K^{*}(0 ; \gamma, \beta)=K^{*}(\gamma, \beta) \quad(0 \leq \gamma, \beta<1) .
\end{gathered}
$$


Corresponding to a conic domain $\Omega_{k, \gamma}$ defined by

$$
\Omega_{k, \gamma}=\left\{u+i v: u>k \sqrt{(u-1)^{2}+v^{2}}+\gamma\right\}
$$

we define the function $q_{k, \gamma}(z)$ which maps $\mathbf{U}$ onto the conic domain $\Omega_{k, \gamma}$ such that $1 \in \Omega_{k, \gamma}$ as the following:

$$
q_{k, \gamma}(z)= \begin{cases}\frac{1+(1-2 \gamma) z}{1-z} & (k=0), \\ \frac{1-\gamma}{1-k^{2}} \cos \left\{\frac{2}{\pi}\left(\cos ^{-1} k\right) i \log \frac{1+\sqrt{z}}{1-\sqrt{z}}\right\}-\frac{k^{2}-\gamma}{1-k^{2}} & (0<k<1), \\ 1+\frac{2(1-\gamma)}{\pi^{2}}\left(\log \frac{1+\sqrt{z}}{1-\sqrt{z}}\right)^{2} & (k=1), \\ \frac{1-\gamma}{k^{2}-1} \sin \left\{\frac{\pi}{2 \zeta(k)} \int_{0}^{\frac{u(z)}{\sqrt{k}}} \frac{d t}{\left.\sqrt{1-t^{2} \sqrt{1-k^{2} t^{2}}}\right\}+\frac{k^{2}-\gamma}{k^{2}-1}}\right. & (k>1) .\end{cases}
$$

Where $u(z)=\frac{z-\sqrt{k}}{1-\sqrt{k} z}$ and $\zeta(k)$ is such that $k=\cosh \frac{\pi \zeta^{\prime}(z)}{4 \zeta(z)}$. By virtue of the properties of the conic domain $\Omega_{k, \gamma}$, we have

$$
\mathfrak{R}\left\{q_{k, \gamma}(z)\right\}>\frac{k+\gamma}{k+1}
$$

Making use of the principal of subordination and the definition of $q_{k, \gamma}(z)$, we may rewrite the subclasses $U S^{*}(k ; \gamma), U C(k ; \gamma), U K(k ; \gamma, \beta)$ and $U K^{*}(k ; \gamma, \beta)$ as the following:

$$
\begin{gathered}
U S^{*}(k ; \gamma)=\left\{f \in \mathbf{A}: \frac{z f^{\prime}(z)}{f(z)} \prec q_{k, \gamma}(z)\right\}, \\
U C(k ; \gamma)=\left\{f \in \mathbf{A}: 1+\frac{z f^{\prime \prime}(z)}{f^{\prime}(z)} \prec q_{k, \gamma}(z)\right\}, \\
U K(k ; \gamma, \beta)=\left\{f \in \mathbf{A}: \exists g \in U S^{*}(k ; \beta) \text { s.t. } \frac{z f^{\prime}(z)}{g(z)} \prec q_{k, \gamma}(z)\right\}, \\
U K^{*}(k ; \gamma, \beta)=\left\{f \in \mathbf{A}: \exists g \in U C(k ; \gamma) \text { s.t. } \frac{\left(z f^{\prime}(z)\right)^{\prime}}{g^{\prime}(z)} \prec q_{k, \gamma}(z)\right\} .
\end{gathered}
$$


We consider the following normalized form

$\mathbf{W}_{\lambda, \mu}(z)=\Gamma(\mu) z \mathbf{W}_{\lambda, \mu}\left(\frac{z}{4}\right)=\sum_{n \geq 0} \frac{\Gamma(\mu) z^{n+1}}{4^{n} n ! \Gamma(\lambda n+\mu)}$,

where $\lambda \geq-1, \mu \in \mathrm{C} \backslash \mathrm{Z}_{0}^{-}, z \in U$. Note that the normalized wright function $\mathbf{w}_{\lambda, \mu}$ was studied recently in [13].

Now, we define an operator $\mathbf{w}_{\lambda, \mu}$ as follows:

$$
\mathbf{w}_{\lambda, \mu} f(z)=\mathbf{w}_{\lambda, \mu}(z) * f(z)=z+\sum_{n \geq 2} \frac{\Gamma(\mu)}{4^{n-1}(n-1) ! \Gamma(\lambda(n-1)+\mu)} a_{n} z^{n}
$$

where $\lambda \geq-1, \mu \in \mathrm{C} \backslash \mathrm{Z}_{0}^{-}, z \in U$. Note that, if $f(z)=\frac{z}{(1-z)}$ then the operator $\mathbf{w}_{\lambda, \mu} f(z)$ reduces to the functions

$$
\mathbf{W}_{v, b, c}(z)=\mathbf{W}_{1, v+\frac{b+1}{2}}(-c z) * \frac{z}{1-z}=(-c) 2^{v} \Gamma(v+(b+1) / 2) z^{1-v / 2} \mathbf{W}_{v, b, c}(\sqrt{z}),
$$

$$
\left.g_{v}(z)=\mathbf{W}_{1, v+1}(-z) * \frac{z}{1-z}=(-1) 2^{v} \Gamma(v+1) / 2\right) z^{1-v / 2} \mathbf{J}_{v}(\sqrt{z})
$$

And

$$
\left.\mathbf{K}_{v}(z)=\mathbf{W}_{1, v+1}\left(\frac{z}{1-z}\right)=\mathbf{W}_{1, v+1}(z) * \frac{z}{1-z}=2^{v} \Gamma(v+1)\right) z^{1-v / 2} \mathbf{I}_{v}(\sqrt{z})
$$

Note that the function $v_{v, b, c}(z)$ was studied recently in $[1,2,11]$ and $g_{v}(z)$ was investigated in $[3,12,14]$.

Corresponding to the function $\mathbf{W}_{\lambda, \mu}(z)$ defined by (1.13), we introduce a function $\mathbf{W}_{\lambda, \mu}^{\alpha}(z)$ given by

$$
\mathbf{w}_{\lambda, \mu}(z) * \mathbf{w}_{\lambda, \mu}^{\alpha}(z)=\frac{z}{(1-z)^{\alpha}} \quad(\alpha>0)
$$

We now define an operator $\mathbf{W}_{\lambda, \mu}^{\alpha} f(z): \mathbf{A} \rightarrow \mathbf{A}$ by

$$
\begin{gathered}
\mathbf{W}_{\lambda, \mu}^{\alpha} f(z)=\mathbf{W}_{\lambda, \mu}^{\alpha}(z) * f(z) \\
\left(\lambda \geq-1, \mu \in \mathrm{C} \backslash \mathrm{Z}_{0}^{-}, \alpha>0 ; z \in U\right) .
\end{gathered}
$$


If $f(z)$ is given by $(1.1)$, then from $(1.17)$, we deduce that

$$
\begin{gathered}
\mathbf{w}_{\lambda, \mu}^{\alpha} f(z)=z+\sum_{n \geq 2} \frac{4^{n-1}(\alpha)_{(n-1)} \Gamma(\lambda(n-1)+\mu)}{\Gamma(\mu)} a_{n} z^{n} \\
\left(\lambda \geq-1, \mu \in \mathrm{C} \backslash \mathrm{Z}_{0}^{-}, \alpha>0 ; z \in U\right) .
\end{gathered}
$$

It is easily to deduce from (1.18) that.

$$
z\left(\mathbf{w}_{\lambda, \mu}^{\alpha} f(z)\right)^{\prime}=\alpha\left(\mathbf{w}_{\lambda, \mu}^{\alpha+1} f(z)\right)-(\alpha-1)\left(\mathbf{w}_{\lambda, \mu}^{\alpha} f(z)\right)
$$

Next, by using the operator $\mathbf{W}_{\lambda, \mu}^{\alpha}$, we introduce the following classes of analytic functions for $\lambda \geq-1, \mu \in \mathrm{C} \backslash \mathrm{Z}_{0}^{-}, \alpha>0, \quad k \geq 0$ and $0 \leq \gamma, \beta<1$ :

$$
\begin{gathered}
U S^{*}(\alpha ; k ; \gamma)=\left\{f \in \mathbf{A}: \mathbf{w}_{\lambda, \mu}^{\alpha} f(z) \in U S^{*}(k ; \gamma)\right\}, \\
U C(\alpha ; k ; \gamma)=\left\{f \in \mathbf{A}: \mathbf{w}_{\lambda, \mu}^{\alpha} f(z) \in U C(k ; \gamma)\right\}, \\
U K(\alpha ; k ; \gamma, \beta)=\left\{f \in \mathbf{A}: \mathbf{w}_{\lambda, \mu}^{\alpha} f(z) \in U K(k ; \gamma, \beta)\right\}, \\
U K^{*}(\alpha ; k ; \gamma, \beta)=\left\{f \in \mathbf{A}: \mathbf{w}_{\lambda, \mu}^{\alpha} f(z) \in U K^{*}(k ; \gamma, \beta)\right\} .
\end{gathered}
$$

We also note that

$$
f(z) \in U S^{*}(\alpha ; k ; \gamma) \Leftrightarrow z f^{\prime}(z) \in U C(\alpha ; k ; \gamma)
$$

and

$$
f(z) \in U K(\alpha ; k ; \gamma, \beta) \Leftrightarrow z f^{\prime}(z) \in U K^{*}(\alpha ; k ; \gamma, \beta)
$$

In this paper, we investigate several inclusion properties of the classes $U S^{*}(\alpha ; k ; \gamma), U C(\alpha ; k ; \gamma)$ , $U K(\alpha ; k ; \gamma, \beta)$ and $U K^{*}(\alpha ; k ; \gamma, \beta)$ associated with the operator $\mathbf{w}_{\lambda, \mu}^{\alpha}$ Some applications involving integral operators are also considered.

\section{Inclusion Properties Involving the Operator $\mathbf{W}_{\lambda, \mu}^{\alpha}$}

In order to prove the main results, we shall need the following lemmas.

Lemma 1 [5]. Let $h(z)$ be convex univalent in $\mathbf{U}$ with $h(0)=1$ and $\mathfrak{R}\{\eta h(z)+\gamma\}>0$ $(\eta, \gamma \in \mathrm{C})$. If $p(z)$ is analytic in $\mathbf{U}$ with $p(0)=1$, then 


$$
p(z)+\frac{z p^{\prime}(z)}{\eta p(z)+\gamma} \prec h(z)
$$

Implies

$$
p(z) \prec h(z) .
$$

Lemma 2 [8]. Let $h(z)$ be convex univalent in $\mathbf{U}$ and let $w$ be analytic in $\mathbf{U}$ with $\mathfrak{R}\{w(z)\} \geq 0$. If $p(z)$ is analytic in $\mathbf{U}$ and $p(0)=h(0)$, then

$$
p(z)+w(z) z p^{\prime}(z) \prec h(z)
$$

Implies

$$
p(z) \prec h(z)
$$

Theorem 1. $U S^{*}(\alpha+1 ; k ; \gamma) \subset U S^{*}(\alpha ; k ; \gamma)$.

Proof. Let $f \in U S^{*}(\alpha+1 ; k ; \gamma)$ and set

$$
p(z)=\frac{z\left(\mathbf{W}_{\lambda, \mu}^{\alpha} f(z)\right)^{\prime}}{\mathbf{W}_{\lambda, \mu}^{\alpha} f(z)} \quad(z \in \mathbf{U}),
$$

where $p(z)$ is analytic in $\mathbf{U}$ with $p(0)=1$. From (1.19) and (2.5), we have

$$
\frac{\mathbf{W}_{\lambda, \mu}^{\alpha+1} f(z)}{\mathbf{W}_{\lambda, \mu}^{\alpha} f(z)}=\frac{1}{\alpha}\{p(z)+(\alpha-1)\} .
$$

Differentiating (2.6) with respect to $z$ and multiplying the result equation by $z$, we obtain

$$
\frac{z\left(\mathbf{W}_{\lambda, \mu}^{\alpha+1} f(z)\right)^{\prime}}{\mathbf{W}_{\lambda, \mu}^{\alpha+1} f(z)}=p(z)+\frac{z p^{\prime}(z)}{p(z)+(\alpha-1)} .
$$

From this and the argument given in Section 1, we may write

$$
p(z)+\frac{z p^{\prime}(z)}{p(z)+(\alpha-1)} \prec q_{k, \gamma}(z) \quad(z \in \mathbf{U}) .
$$

Since $(\alpha-1)>0$ and $\mathfrak{R}\left\{q_{k, \gamma}(z)\right\}>\frac{k+\gamma}{k+1}$, we see that 


$$
\mathfrak{R}\left\{q_{k, \gamma}(z)+(\alpha-1)\right\}>0 \quad(z \in \mathbf{U}) .
$$

Applying Lemma 1 to (2.8), it follows that $p(z) \prec q_{k, \gamma}(z)$, that is, $f \in U S^{*}(\alpha ; k ; \gamma)$.

Theorem 2. $U C(\alpha+1 ; k ; \gamma) \subset U C(\alpha ; k ; \gamma)$.

Proof. Applying (1.24) and Theorem 1, we observe that

$$
\begin{aligned}
f(z) & \in U C(\alpha+1 ; k ; \gamma) \Leftrightarrow z f^{\prime}(z) \in U S^{*}(\alpha+1 ; k ; \gamma) \\
& \Rightarrow z f^{\prime}(z) \in U S^{*}(\alpha ; k ; \gamma) \\
& \Leftrightarrow f(z) \in U C(\alpha ; k ; \gamma),
\end{aligned}
$$

which evidently proves Theorem 2 .

Theorem 3. $U K(\alpha+1 ; k ; \gamma, \beta) \subset U K(\alpha ; k ; \gamma, \beta)$.

Proof. Let $f \in U K(\alpha+1 ; k ; \gamma, \beta)$. Then, from the definition of $U K(\alpha+1 ; k ; \gamma, \beta)$, there exists a function $r(z) \in U S^{*}(k ; \gamma)$ such that

$$
\frac{z\left(\mathbf{W}_{\lambda, \mu}^{\alpha+1} f(z)\right)^{\prime}}{r(z)} \prec q_{k, \gamma}(z) .
$$

Choose the function $g(z)$ such that $\mathbf{w}_{\lambda, \mu}^{\alpha+1} g(z)=r(z)$. Then, $g \in U S^{*}(\alpha+1 ; k ; \gamma)$ and

$$
\frac{z\left(\mathbf{W}_{\lambda, \mu}^{\alpha+1} f(z)\right)^{\prime}}{\mathbf{w}_{\lambda, \mu}^{\alpha+1} g(z)} \prec q_{k, \gamma}(z) .
$$

Now let

$$
p(z)=\frac{z\left(\mathbf{w}_{\lambda, \mu}^{\alpha} f(z)\right)^{\prime}}{\mathbf{w}_{\lambda, \mu}^{\alpha} g(z)}
$$

where $p(z)$ is analytic in $\mathbf{U}$ with $p(0)=1$. Since $g \in U S^{*}(\alpha+1 ; k ; \gamma)$, by Theorem 1 , we know that $g \in U S^{*}(\alpha ; k ; \gamma)$. Let

$$
t(z)=\frac{z\left(\mathbf{W}_{\lambda, \mu}^{\alpha} g(z)\right)^{\prime}}{\mathbf{w}_{\lambda, \mu}^{\alpha} g(z)} \quad(z \in \mathbf{U}),
$$

where $t(z)$ is analytic in $\mathbf{U}$ with $\mathfrak{R}\{t(z)\}>\frac{k+\gamma}{k+1}$. Also, from (2.13), we note that

$$
z\left(\mathbf{w}_{\lambda, \mu}^{\alpha} f(z)\right)^{\prime}=\mathbf{w}_{\lambda, \mu}^{\alpha} z f^{\prime}(z)=\left(\mathbf{w}_{\lambda, \mu}^{\alpha} g(z)\right) p(z)
$$


Differentiating both sides of (2.14) with respect to $z$ and multiplying the result equation by $z$, we obtain

$$
\frac{z\left(\mathbf{W}_{\lambda, \mu}^{\alpha} z f^{\prime}(z)\right)^{\prime}}{\mathbf{w}_{\lambda, \mu}^{\alpha} g(z)}=\frac{z\left(\mathbf{w}_{\lambda, \mu}^{\alpha} g(z)\right)^{\prime}}{\mathbf{w}_{\lambda, \mu}^{\alpha} g(z)} p(z)+z p^{\prime}(z)=t(z) p(z)+z p^{\prime}(z) .
$$

Now using the identity (1.19) and (2.15), we obtain

$$
\begin{aligned}
& \frac{z\left(\mathbf{w}_{\lambda, \mu}^{\alpha+1} f(z)\right)^{\prime}}{\mathbf{w}_{\lambda, \mu}^{\alpha+1} g(z)}=\frac{\mathbf{w}_{\lambda, \mu}^{\alpha+1} z f^{\prime}(z)}{\mathbf{w}_{\lambda, \mu}^{\alpha+1} g(z)}=\frac{z\left(\mathbf{w}_{\lambda, \mu}^{\alpha} z f^{\prime}(z)\right)^{\prime}+(\alpha-1) \mathbf{w}_{\lambda, \mu}^{\alpha} z f^{\prime}(z)}{z\left(\mathbf{w}_{\lambda, \mu}^{\alpha} g(z)\right)^{\prime}+(\alpha-1) \mathbf{w}_{\lambda, \mu}^{\alpha} g(z)} \\
& =\frac{\frac{z\left(\mathbf{w}_{\lambda, \mu^{\alpha}}^{\alpha} z f^{\prime}(z)\right)^{\prime}}{\mathbf{w}_{\lambda, \mu}^{\alpha} g(z)}+(\alpha-1) \frac{z\left(\mathbf{w}_{\lambda, \mu}^{\alpha} f(z)\right)^{\prime}}{\mathbf{w}_{\lambda, \mu}^{\alpha} g(z)}}{\frac{z\left(\mathbf{w}_{\lambda, \mu}^{\alpha} g(z)\right)^{\prime}}{\mathbf{w}_{\lambda, \mu}^{\alpha} g(z)}+(\alpha-1)} \\
& =\frac{t(z) p(z)+z p^{\prime}(z)+(\alpha-1) p(z)}{t(z)+(\alpha-1)}
\end{aligned}
$$

$$
=p(z)+\frac{z p^{\prime}(z)}{t(z)+(\alpha-1)}
$$

Since $(\alpha-1)>0$ and $\mathfrak{R}\{t(z)\}>\frac{k+\gamma}{k+1}$, we see that

$$
\mathfrak{R}\{t(z)+(\alpha-1)\}>0 \quad(z \in \mathbf{U})
$$

Hence, applying Lemma 2, we can show that $p(z) \prec q_{k, \gamma}(z)$ so that $f \in U K(\alpha ; k ; \gamma, \beta)$. This completes the proof of Theorem 3.

Theorem 4. $U K^{*}(\alpha+1 ; k ; \gamma, \beta) \subset U K^{*}(\alpha ; k ; \gamma, \beta)$.

Proof. Just as we derived Theorem 2 as consequence of Theorem 1 by using the equivalence (1.24), we can also prove Theorem 4 by using Theorem 3 and the equivalence (1.25).

\section{Inclusion Properties Involving the Integral Operator $F_{c}$}

In this section, we consider the generalized Libera integral operator $F_{c}(\operatorname{see}[4,6,7])$ defined by 


$$
F_{c}(f)(z)=\frac{c+1}{z^{c}} \int_{0}^{z} t^{c-1} f(t) d t \quad(f \in \mathbf{A} ; c>-1)
$$

Theorem 5. Let $c>-\frac{k+\gamma}{k+1}$. If $f \in U S^{*}(\alpha ; k ; \gamma)$, then $F_{c}(f) \in U S^{*}(\alpha ; k ; \gamma)$.

Proof. Let $f \in U S^{*}(\alpha ; k ; \gamma)$ and set

$$
p(z)=\frac{z\left(\mathbf{w}_{\lambda, \mu}^{\alpha} F_{c}(f)(z)\right)^{\prime}}{\mathbf{w}_{\lambda, \mu}^{\alpha} F_{c}(f)(z)}(z \in \mathbf{U})
$$

where $p(z)$ is analytic in $\mathbf{U}$ with $p(0)=1$. From (3.2), we have

$$
z\left(\mathbf{w}_{\lambda, \mu}^{\alpha} F_{c}(f)(z)\right)^{\prime}=(c+1) \mathbf{w}_{\lambda, \mu}^{\alpha} f(z)-c \mathbf{w}_{\lambda, \mu}^{\alpha} F_{c}(f)(z)
$$

Then, by using (3.2) and (3.3), we obtain

$$
(c+1) \frac{\mathbf{W}_{\lambda, \mu}^{\alpha} f(z)}{\mathbf{W}_{\lambda, \mu}^{\alpha} F_{c}(f)(z)}=p(z)+c .
$$

Taking the logarithmic differentiation on both sides of (3.4) and multiplying by $z$, we have

$$
p(z)+\frac{z p^{\prime}(z)}{p(z)+c}=\frac{z\left(\mathbf{w}_{\lambda, \mu}^{\alpha} f(z)\right)^{\prime}}{\mathbf{w}_{\lambda, \mu}^{\alpha} f(z)} \prec q_{k, \gamma}(z) .
$$

Hence, by virtue of Lemma 1 , we conclude that $p(z) \prec q_{k, \gamma}(z)$ in $\mathbf{U}$, which implies that $F_{c}(f) \in U S^{*}(\alpha ; k ; \gamma)$.

Theorem 6. Let $c>-\frac{k+\gamma}{k+1}$. If $f \in U C(\alpha ; k ; \gamma)$, then $F_{c}(f) \in U C(\alpha ; k ; \gamma)$.

Proof. By applying Theorem 5, it follows that

$$
\begin{aligned}
f(z) & \in U C(\alpha ; k ; \gamma) \Leftrightarrow z f^{\prime}(z) \in U S^{*}(\alpha ; k ; \gamma) \\
& \left.\Rightarrow F_{c}\left(z f^{\prime}\right)(z) \in U S^{*}(\alpha ; k ; \gamma) \quad \text { (by Theorem } 5\right) \\
\Leftrightarrow z\left(F_{c}(f)(z)\right) & \in U S^{*}(\alpha ; k ; \gamma)
\end{aligned}
$$

$$
\Leftrightarrow F_{c}(f)(z) \in U C(\alpha ; k ; \gamma)
$$

which proves Theorem 6 . 
Theorem 7. Let $c>-\frac{k+\gamma}{k+1}$. If $f \in U K(\alpha ; k ; \gamma, \beta)$, then $F_{c}(f) \in U K(\alpha ; k ; \gamma, \beta)$.

Proof. Let $f \in U K(\alpha ; k ; \gamma, \beta)$. Then, in view of the definition of the class $U K(\alpha ; k ; \gamma, \beta)$, there exists a function $g \in U S^{*}(\alpha ; k ; \gamma)$ such that

$$
\frac{z\left(\mathbf{w}_{\lambda, \mu}^{\alpha} f(z)\right)^{\prime}}{\mathbf{w}_{\lambda, \mu}^{\alpha} g(z)} \prec q_{k, \gamma}(z) .
$$

Thus, we set

$$
p(z)=\frac{z\left(\mathbf{W}_{\lambda, \mu}^{\alpha} F_{c}(f)(z)\right)^{\prime}}{\mathbf{W}_{\lambda, \mu}^{\alpha} F_{c}(g)(z)} \quad(z \in \mathbf{U})
$$

where $p(z)$ is analytic in $\mathbf{U}$ with $p(0)=1$. Since $g \in U S^{*}(\alpha ; k ; \gamma)$, we see from Theorem 5 . that $F_{c}(g) \in U S^{*}(\alpha ; k ; \gamma)$. Using (3.3) and let

$$
t(z)=\frac{z\left(\mathbf{W}_{\lambda, \mu}^{\alpha} F_{c}(g)(z)\right)^{\prime}}{\mathbf{W}_{\lambda, \mu}^{\alpha} F_{c}(g)(z)},
$$

where $t(z)$ is analytic in $\mathbf{U}$ with $\mathfrak{R}\{t(z)\}>\frac{k+\gamma}{k+1}$. Using (3.8), we have

$$
\mathbf{w}_{\lambda, \mu}^{\alpha} z F_{c}^{\prime}(f)(z)=\left(\mathbf{W}_{\lambda, \mu}^{\alpha} F_{c}(g)(z)\right) p(z)
$$

Differentiating both sides of (3.10) with respect to $z$ and multiplying by $z$, we obtain

$$
\frac{z\left(\mathbf{w}_{\lambda, \mu}^{\alpha} z F_{c}^{\prime}(f)(z)\right)^{\prime}}{\mathbf{w}_{\lambda, \mu}^{\alpha} F_{c}(g)(z)}=\frac{z\left(\mathbf{w}_{\lambda, \mu}^{\alpha} F_{c}(f)(z)\right)^{\prime}}{\mathbf{w}_{\lambda, \mu}^{\alpha} F_{c}(g)(z)} p(z)+z p^{\prime}(z)
$$

$$
=t(z) p(z)+z p^{\prime}(z)
$$


Now using the identity (3.3) and (3.11), we obtain

$$
\begin{aligned}
\frac{z\left(\mathbf{W}_{\lambda, \mu}^{\alpha} f(z)\right)^{\prime}}{\mathbf{W}_{\lambda, \mu}^{\alpha} g(z)} & =\frac{\mathbf{W}_{\lambda, \mu}^{\alpha} z f^{\prime}(z)}{\mathbf{W}_{\lambda, \mu}^{\alpha} g(z)}=\frac{z\left(\mathbf{w}_{\lambda, \mu}^{\alpha} z F_{c}^{\prime}(f)(z)\right)^{\prime}+c \mathbf{W}_{\lambda, \mu}^{\alpha} z F_{c}^{\prime}(f)(z)}{z\left(\mathbf{w}_{\lambda, \mu}^{\alpha} F_{c}(g)(z)\right)^{\prime}+c \mathbf{W}_{\lambda, \mu}^{\alpha} F_{c}(g)(z)} \\
& =\frac{\frac{z\left(\mathbf{W}_{\lambda, \mu}^{\alpha} z F_{c}^{\prime}(f)(z)\right)^{\prime}}{\mathbf{W}_{\lambda, \mu}^{\alpha} F_{c}(g)(z)}+c \frac{z\left(\mathbf{W}_{\lambda, \mu}^{\alpha} F_{c}(f)(z)\right)^{\prime}}{\mathbf{w}_{\lambda, \mu}^{\alpha} F_{c}(g)(z)}}{\frac{z\left(\mathbf{W}_{\lambda, \mu}^{\alpha} F_{c}(g)(z)\right)^{\prime}}{\mathbf{W}_{\lambda, \mu}^{\alpha} F_{c}(g)(z)}+c} \\
& =\frac{t(z) p(z)+z p^{\prime}(z)+c p(z)}{t(z)+c}=p(z)+\frac{z p^{\prime}(z)}{t(z)+c}
\end{aligned}
$$

Since $c>-\frac{k+\gamma}{k+1}$ and $\mathfrak{R}\{t(z)\}>\frac{k+\gamma}{k+1}$, we see that

$$
\mathfrak{R}\{t(z)+c\}>0 \quad(z \in \mathbf{U})
$$

Applying Lemma 2 to (3.12), it follows that $p(z) \prec q_{k, \gamma}(z)$, that is $F_{c}(f) \in U K(\alpha ; k ; \gamma, \beta)$.

Theorem 8. Let $c>-\frac{k+\gamma}{k+1}$. If $f \in U K^{*}(\alpha ; k ; \gamma, \beta)$, then $F_{c}(f) \in U K^{*}(\alpha ; k ; \gamma, \beta)$.

Proof. Just as we derived Theorem 6 as consequence of Theorem 5 and (1.24), we easily deduce the integral-preserving property asserted by Theorem 8 by using Theorem 7 and (1.25).

\section{Reference}

[1] A. Baricz, Geometric properties of generalized Bessel functions, Publ. Math. Debrecen. 73 (2008), $155-178$.

[2] A. Baricz and S. Ponnusamy, Starlikeness and convexity of generalized Bessel functions, Integral Transforms Spec. Funct. 21(2010), no. 9, 641-653.

[3] A. Baricz, P. A. Kup an and R. Sz asz, The radius of starlikeness of normalized Bessel functions of the rst kind, Proc. Amer. Math. Soc. 142(2014), no. 6, 2019-2025.

[4] S. D. Bernardi, Convex and univalent functions, Trans. Amer. Math. Soc., 135(1996), 429-446.

[5] P. Eenigenburg, S. S. Miller, P. T. Mocanu, and M. O. Reade, On a Briot-Bouquet differential subordination, in General Inequalities, 3 (Oberwolfach, 1981), vol. 64 of Internationale Schriftenreihe zur Numerischen Mathematik, pp. 339--348, Birkhäuser, Basel, Switzerland, 1983.

[6] R. J. Libera, Some classes of regular univalent functions, Proc. Amer. Math. Soc., 16(1965), 755-658.

[7] A. E. Livingston, On the radius of univalence of certain analytic functions, Proc. Amer. Math. Soc., 17 (1966), 352-357. 
[8] S. S.Miller and P. T.Mocanu, Differential subordinations and univalent functions, Michigan Math. J., 28(1981), no. 2, 157--172.

[9] S. S. Miller and P. T. Mocanu, Differential Subordinations: Theory and Applications, Series on Monographs and Textbooks in Pure and Applied Mathematics, Vol. 225, Marcel Dekker, New York and Basel, 2000.

[10] S. Miller and P. T. Mocanu, Subordinants of differential superordinations, Complex Variables Theory Appl., 48(2003), no.10, 815--826.

[11] S. R. Mondal and A. Swaminathan, Geometric properties of generalized Bessel functions,Bull. Malays. Math. Soc. (2) 35(2012), no. 1, 179-194.

[12] J. K. Prajapat, Certain geometric properties of normalized Bessel functions, Appl. Math.Lett. 24(2011), no. 12, 2133-2139.

[13] J. K. Prajapat, Certain geometric properties of the Wright functions, Integral Transforms Spec. Funct. 26(2015), no. 3, 203-212.

[14] R. Szasz and P. A. Kupan, About the univalence of the Bessel functions, Stud. Univ. Babes-Bolyai Math. 54(2009), no. 1, 127-132.

\footnotetext{
*Corresponding author.

E-mail address: ekram_008eg@yahoo.com
} 